\title{
Social Network Analysis and Its Applications for Authors and Keywords in the JKSS
}

\author{
Jong Goen Kim ${ }^{a}$, Soon-Kuek Choi ${ }^{b}$, Yong-Seok Choi ${ }^{1, b}$ \\ ${ }^{a}$ School of Computer and Information, Busan Institute of Science and Technology \\ ${ }^{b}$ Department of Statistics, Pusan National University
}

\begin{abstract}
Social network analysis is a graphical technique to search the relationships and characteristics of nodes (people, companies, and organizations) and an important node for positioning a visualized social network figure; however, it is difficult to characterize nodes in a social network figure. Therefore, their relationships and characteristics could be presented through an application of correspondence analysis to an affiliation matrix that is a type of similarity matrix between nodes. In this study, we provide the relationships and characteristics around authors and keywords in the JKSS(Journal of the Korean Statistical Society) of the Korean Statistical Society through the use of social network analysis and correspondence analysis.
\end{abstract}

Keywords: Social network analysis, centrality, correspondence analysis, Journal of the Korean Statistical Society.

\section{1. 서론}

우리는 처음 만나는 사람인데 알고 보니 친구의 친구처럼 가까운 사이일 경우 세상이 참 좁다는 말 을 사용한다 (Huh, 2010). 실제로 1967년에 사회학자 Stanley Milgram이 주장한 6단계 분리(six degrees of separation)를 거치면 이 세상의 모두가 서로 아는 사람으로 연결될 수 있다고 했다. 현대사회 는 이전 사회보다 인터넷과 스마트폰 등과 같은 각종 통신기술의 발달로 인하여 이 이론에서 주장하는 것보다 사람들의 관계가 더욱더 긴밀하게 이루어지고 있다. 오늘날에는 사회학을 비롯하여 정치학, 경 영학, 공학 등의 분야에서 관계의 중요성이 인식되면서 이에 대한 분석이 필요하게 되었다.

사회네트워크 분석(social network analysis; SNA)은 Barnes (1954)에 의해 처음 사용된 용어로, 사 회네트워크 현상에서 관계 구조를 시각화하여 중요한 위치에 있는 사람, 기업, 기관 등을 찾아내어 다 양한 영역에 적용시킬 수 있는 분석기법이다 (Sohn, 2002). 최근 이 분석을 응용한 사례를 살펴보면, Jung 과 Chung (2008)은 대한예방의학회지 게재논문을 분석하여 공저자.핵심어 네트워크와 군집 양상 을 살펴보았고, $\mathrm{Kim}$ 등 (2009)은 충성고객과 이탈고객의 구매 특성을 비교 연구 하였다. 그리고 Choi 등 (2011)은 축구경기 분석을 하였다.

Korean Statistical Society (2011)는 한국통계학회 학술지인 JKSS를 일정 주제로 분류하여 각 주 제에 따른 논문과 저자에 대한 빈도를 살펴보았다. 그러나 본 연구에서는 이러한 사회네트워크 분 석을 활용하여 단순히 빈도뿐만 아니라, JKSS의 SCIE(Science Citation Index Expanded)급 전환 이전

This work was supported by the Financial Supporting Project of Long-term Overseas Dispatch of PNU's Tenure-track Faculty, 2011.

${ }^{1}$ Corresponding author: Professor, Department of Statistics, Pusan National University, Busan 609-735, Korea.

E-mail: yschoi@pusan.ac.kr 
과 이후의 특징을 살펴보려 한다. 2절에서는 사회네트워크 분석과 이 분석에서 중요한 대상의 인접행 렬(adjacency matrix)을 다변량 그래프적으로 분석하기 위한 대응분석(correspondence analysis)의 기초 이론에 대해 설명하고, 3 절에서는 2절의 기법을 활용하여 $\mathrm{JKSS}$ 의 특징을 살펴본 후, 끝으로 4절에서 본 연구를 정리·요약하려 한다.

\section{2. 사회네트워크 분석 및 대응분석}

2.1절에서는 Sohn (2002, 5장)과 Huh (2010, 1장과 2장)를 참고하여 사회네트워크 분석의 정의와 자료의 구조에 대해서 알아보고, 2.2절에서는 이 분석을 통하여 형상화된 네트워크의 특징을 파악하기 위한 기법인 대응분석을 Choi (2001, 2장)를 참고하여 소개하려 한다.

\section{1. 사회네트워크 분석}

사회네트워크 분석에서는 사회적 구조를 간단하게 노드(node)와 선(link)으로 표현하는데, 노드는 개인, 사회, 집단, 조직 등으로 정의되고, 선은 이들의 정보거래, 친구관계, 직무거래 등 다양한 관계로 정의된다. 네트워크에서는 중요한 노드 또는 네트워크 흐름에 중요한 역할을 하는 노드를 찾는 것이 중요하며 이를 나타내는 측도로 중심성(centrality)이 사용된다.

각각의 노드는 인접행렬을 통해 행과 열로 나타낼 수 있고, 행과 열이 교차되는 셀은 관계(선)가 있 으면 1 , 없으면 0 의 값을 가질 수 있다. 즉 $q$ 개의 노드를 가진 인접행렬을 $A=\left\{a_{i j}\right\}, i, j=1,2, \ldots, q$ 라 할때, $a_{i j}=1$ 이면 노드 $i$ 에서 노드 $j$ 로 가는 관계가 있음을 의미한다. 인접행렬은 행과 열이 동일 대상 을 나타내지만 다른 대상일 경우 $n$ 개의 행 노드와 $m$ 개의 열 노드의 연결로 교차자료가 구성되고, 이를 $n \times m$ 연관행렬(affiliation matrix)이라고 한다.

일반적인 수치자료에서 평균 등의 중심위치 파악이 중요한 것처럼 사회네트워크 분석에서도 중심 노드를 이용하여 각 네트워크의 중심을 파악한다. 중심노드는 중심성이 높은것을 말하며 네트워크에 서 핵심적인 위치에 있으면서 네트워크의 흐름에 주요한 역할을 하며 정보 교류의 핵심역할을 한다. 중심성을 나타내는 측도는 근접 중심성(closeness centrality)과 매개 중심성(betweenness centrality)으 로 나눌 수 있다.

먼저 근접 중심성은 하나의 노드에서 직접 연결된 노드뿐만 아니라 간접적으로 연결된 모든 노드들 의 거리를 산출하여 각 노드마다 전체 네트워크에서 중심에 얼마나 가까이 위치하고 있는지를 측정하 는 방법이다. $n$ 개의 노드로 구성된 네트워크에서 $d(i, j)$ 를 노드 $i$ 에서 노드 $j$ 에 이르는 거리라고 할 때, $i$ 번째 노드에 대한 일반화 근접 중심성 $C_{C}(i)$ 는

$$
C_{C}(i)=\frac{\sum_{j \neq i}\left(\frac{1}{d(i, j)}\right)}{n-1}, \quad i=1,2, \ldots, n
$$

와 같고, 일반화 근접 중심성의 범위는 0 과 1 사이다. 본 연구에서는 일반화 근접 중심성이 높을 수록 논문에서 중요한 핵심어이다.

다음으로 매개 중심성은 네트워크 내에서 한 노드가 담당하는 매개자 혹은 중재자 역할의 정도를 측정한 방법이다. 예를 들어 노드 $\mathrm{A}$ 와 $\mathrm{B}$ 가 오직 노드 $\mathrm{C}$ 를 통해서만 관계를 맺을 수 있는 경우 노드 $\mathrm{C}$ 는 높은 매개 중심성을 갖는다고 할 수 있다. 네트워크 내에서 $g_{i j}$ 를 노드 $i$ 와 노드 $j$ 사이에 존재하는 최단 거리 경로의 경우의 수라 하고, $g_{i k j}$ 를 노드 $i$ 와 노드 $j(i \neq j)$ 사이에 존재하는 노드 $k$ 를 경유하는 
횟수라 할 때, 노드 $k$ 에 대한 일반화 매개 중심성 $C_{B}(k)$ 는

$$
C_{B}(k)=\frac{\sum_{i \neq k} \sum_{j \neq k, \neq i}\left(\frac{g_{i k j}}{g_{i j}}\right)}{\left[\frac{(n-2)(n-1)}{2}\right]}, \quad k=1,2, \ldots, n
$$

와 같고, 일반화 매개 중심성의 범위는 0 과 1 사이다. 본 연구에서는 일반화 매개 중심성이 높을 수록 여러 논문들을 이어주는 영향력이 큰 핵심어의 역할을 한다.

\section{2. 대응분석}

사회네트워크 분석은 네트워크 그림을 통하여 노드들 간의 연결적 관계만을 파악할 수 있으나, 그 들간의 특징을 구체적으로 파악하기 힘들다는 단점을 가지고 있다. 이를 보완하기 위하여 다변량 기법 인 대응분석을 적용하고자 한다. 대응분석이란 분할표 자료의 행과 열을 저차원 공간상의 점들로 동시 에 나타내어 그들의 관계를 탐구하려는 탐색적 자료분석기법이다.

행의 수가 $p$ 이고 열의 수가 $q(\leq p)$ 인 이원분할표 자료행렬 $\mathbf{O}=\left\{o_{i j}\right\}, i=1,2, \ldots, p ; j=1,2, \ldots, q$ 를 2.1절에서 정의한 연관행렬이라 하자. 연관행렬 $\mathbf{O}$ 로부터 행렬교적 $\mathbf{O}^{\prime} \mathbf{O}$ 는 열에 관한 인접행렬이 고, $\mathbf{O O}^{\prime}$ 은 행에 관한 인접행렬이 된다. 이와 같은 연관행렬 $\mathbf{O}$ 를 이용하여 대응분석을 적용하기 위한 대응행렬(correspondence matrix)은

$$
\mathbf{F}=\left\{f_{i j}\right\}, \quad f_{i j}=\frac{o_{i j}}{o_{++}}
$$

이며 $o_{++}$는 $\mathbf{O}$ 의 모든 원소들의 전체합이 된다.

대응분석에서 다차원의 정보를 저차원으로 표현하기 위한 대응분석도의 좌표행렬을 얻기 위해 중 심화(centred) 대응행렬 $\mathbf{F}-\mathbf{r c}^{\prime}$ 의 일반화 비정칙치분해

$$
\mathbf{F}-\mathbf{r c}^{\prime}=\mathbf{U} \mathbf{D}_{\lambda} \mathbf{V}^{\prime}
$$

를 이용한다. 여기서 $\mathbf{r}=\left(f_{1+}, \ldots, f_{p+}\right)^{\prime}$ 과 $\mathbf{c}=\left(f_{+1}, \ldots, f_{+q}\right)^{\prime}$ 는 크기가 $p \times 1$ 과 $q \times 1$ 인 벡터이며, $f_{i_{+}}=$ $\sum_{j=1}^{q} f_{i j}$ 과 $f_{+j}=\sum_{i=1}^{p} f_{i j}$ 이다. 그리고 $\mathbf{D}_{\lambda}=\operatorname{diag}\left(\lambda_{1}, \ldots, \lambda_{q-1}\right)$ 는 비정칙치를 대각원소로 하는 대각행렬 이고, $\mathbf{U}$ 와 $\mathbf{V}$ 는 $\mathbf{U}^{\prime} \mathbf{D}_{r}^{-1} \mathbf{U}=\mathbf{V}^{\prime} \mathbf{D}_{c}^{-1} \mathbf{V}=\mathbf{I}$ 를 만족하며, $\mathbf{D}_{r}=\operatorname{diag}\left(f_{1^{+}}, \ldots, f_{p^{+}}\right)$과 $\mathbf{D}_{c}=\operatorname{diag}\left(f_{+1}, \ldots, f_{+q}\right)$ 는 크기가 $p \times p$ 와 $q \times q$ 인 대각행렬이다.

식 (2.4)로부터 대응분석도를 위한 행 좌표행렬은 $\mathbf{D}_{r}^{-1} \mathbf{U} \mathbf{D}_{\lambda}$ 이고, 열 좌표행렬은 $\mathbf{D}_{c}^{-1} \mathbf{U} \mathbf{D}_{\lambda}$ 이며, $s(\leq$ $q-1)$ 차원의 대응분석도의 설명력은 $\left(\sum_{k=1}^{s} \lambda_{k}^{2} / \sum_{k=1}^{q-1} \lambda_{k}^{2}\right) \times 100 \%$ 이다.

\section{3. 활용 사례}

\section{1. 연구 자료 및 방법}

본 연구에서는 JKSS의 게재 논문들의 특징을 살펴보기 위해 2003년(32권 2호)부터 2010년(39권 4 호)까지의 논문들의 정보를 사용하였다. 이 중 2008 년 37 권 1 호의 경우 핵심어가 명시되지 않아 분석 자료에서 제외하였다. 이는 2003년 이전의 자료에서는 각 논문의 핵심어(key-word)가 명시되지 않았 고, 2008년 이후부터 JKSS 가 SCIE급의 학술지로 변경되면서 SCIE급 전환 이전과 이후의 변화를 살펴 보기 용이할 것이라 판단되어 2003년부터 2010년까지 자료를 수집하였다. 따라서 연구 자료는 Table 1 과 같이 278 편의 논문, 413 명의 저자, 1,059 개의 핵심어로 구성되어 있다. 
Table 1: Research

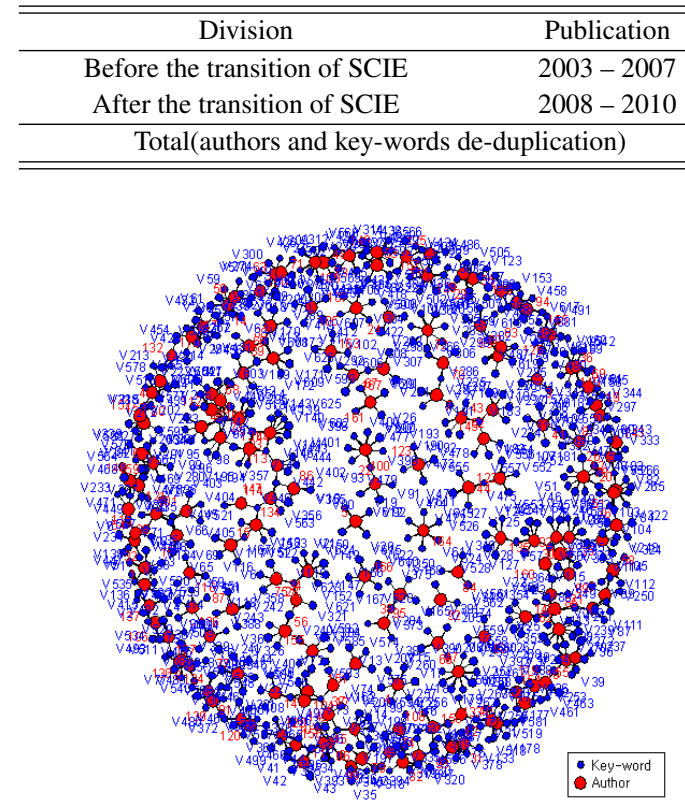

(a) The entire association networks

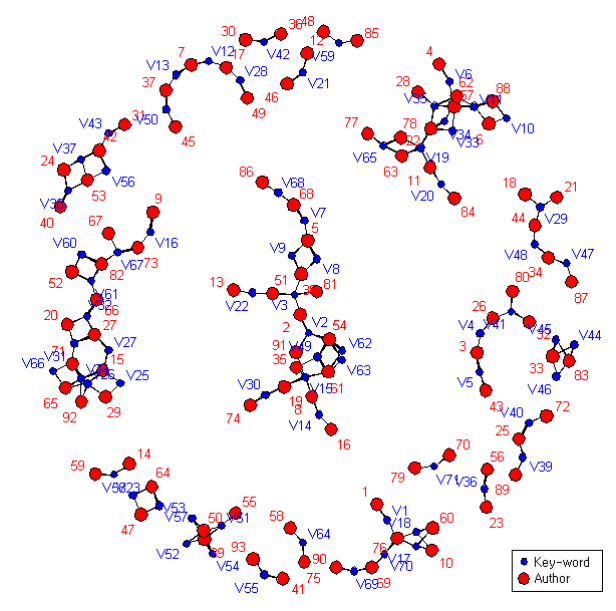

(b) The modified association networks

Figure 1: Papers and key-words associated with the networks before the transition of SCIE

노드는 논문, 핵심어, 저자이고 선은 논문에 핵심어가 포함되어 있으면 관계가 있다고 보고 1 , 포함 되어있지 않으면 관계가 없다고 보고 0 으로 표현하였다. 일반적으로 한 편의 논문은 여러 가지 핵심어 들을 이용하여 작성되고, 핵심어들의 중요도는 파악하기 힘들기 때문에 일정하게 가중치를 1 로 부여하 였다.

\section{2. $\mathrm{SCIE}$ 급 전환 이전의 $\mathrm{JKSS}$}

Table 1 의 SCIE급 전환 이전의 JKSS를 살펴보면 167 편의 논문, 235 명의 저자, 626 개의 핵심어로 구성되어 있다. Figure 1의 (a)는 논문과 핵심어에 대한 $167 \times 626$ 의 전체 연관네트워크 그림이다. 그 림을 살펴보면 방대한 양의 핵심어의 사용으로 인하여 구체적인 정보를 확인하기 힘들기 때문에 626 개 의 핵심어 중에서 최소 2 편 이상의 논문에서 제시된 핵심어를 선별하였고, 선별된 71 개의 핵심어가 사 용된 93 편의 논문, 139 명의 저자로 수정된 자료에 대한 분석을 실시하였다. Figure 1 의 (b)는 수정된 $93 \times 71$ 의 연관네트워크 그림이다.

수정된 연관네트워크로부터 핵심어간의 특징을 살펴보기 위해 행렬교적을 이용한 $71 \times 71$ 인접행 렬의 핵심어 네트워크는 Figure 2와 같다. 이 그림을 보면 크게 세 그룹의 네트워크가 형성되고 나머지 핵심어들은 독립적이거나, 작은 네트워크를 형성하는 것을 알 수 있다. 식 (2.1)에 의한 근접 중심성과 식 (2.2)에 의한 매개 중심성의 값을 기준으로 상위 $10 \%$ 의 노드에 대한 정보는 Table 2 와 같다. 가장 높 은 근접 중심성을 보이는 reference prior(V26)를 중심으로 matching prior(V24) 등이 그룹 B의 핵심어 네트워크를 구성하고, $\mathrm{Kim}$ 등 (2003), Kim 등 (2006), Heo와 Kim (2007) 등 10편의 논문에서 핵심어를 공유하고 있다. 그룹 $\mathrm{B}$ 에서 매개 중심성이 가장 높은 핵심어는 metropolis-hastings algorithm(V32)이 고, markov chain monte carlo(V61), fractional bayes factor(V27), gibbs sampler(V31)가 매개 중심성이 


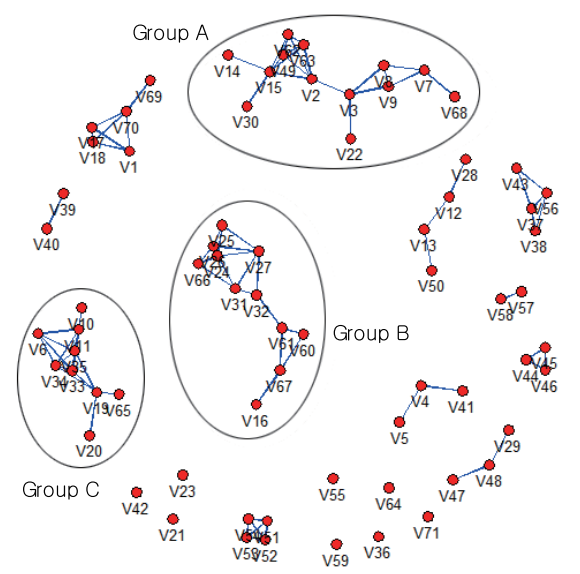

Figure 2: Key-words network before the transition of SCIE

Table 2: Key-words network centrality before the transition of SCIE

\begin{tabular}{lccc|lccc}
\hline \hline \multicolumn{1}{c}{ Key-word } & Node & $\begin{array}{c}\text { Closeness } \\
\text { centrality }\end{array}$ & Group & \multicolumn{1}{c}{ Key-word } & Node & $\begin{array}{c}\text { Betweenness } \\
\text { centrality }\end{array}$ & Group \\
\hline reference prior & V26 & 0.1701 & B & efficiency & V3 & 0.0323 & A \\
matching prior & V24 & 0.1606 & B & consistency & V2 & 0.0298 & A \\
extreme value index & V49 & 0.1469 & A & metropolis-hastings algorithm & V32 & 0.0199 & B \\
rate of convergence & V35 & 0.1452 & C & asymptotic normality & V15 & 0.0174 & A \\
asymptotic normality & V15 & 0.1279 & A & markov chain monte carlo & V61 & 0.0174 & B \\
second order parameter & V62 & 0.1279 & A & weak convergence & V19 & 0.0108 & C \\
extreme value distribution & V63 & 0.1279 & A & coefficient of variation & V7 & 0.0091 & A \\
jump size & V33 & 0.1262 & C & fractional bayes factor & V27 & 0.0086 & B \\
one-side kernel & V34 & 0.1262 & C & gibbs sampler & V31 & 0.0086 & B \\
\hline \hline
\end{tabular}

Table 3: Before the transition of SCIE center of a group of key-words and papers

\begin{tabular}{cll}
\hline \hline Group & \multicolumn{1}{c}{ Division } & \multicolumn{1}{c}{ Key-words and papers } \\
\hline \multirow{2}{*}{ A } & Closeness centrality & extreme value index(V49), asymptotic normality(V15) \\
& Betweenness centrality & efficiency(V3), consistency(V2), asymptotic normality(V15) \\
& Sharing papers & Yun (2005), Yun (2006) and 11 papers \\
\hline \multirow{3}{*}{ B } & Closeness centrality & reference prior(V26), matching prior(V24) \\
& Betweenness centrality & metropolis-hastings algorithm(V32), markov chain monte carlo(V61) \\
& Sharing papers & Kim et al. (2003), Kim et al. (2006), Heo and Kim (2007) and 10 papers \\
\hline \multirow{2}{*}{ C } & Closeness centrality & rate of convergence(V35), jump size(V33), one-side kernel(V34) \\
& Betweenness centrality & weak convergence(V19) \\
& Sharing papers & Huh (2004), Kang and Huh (2006) and 10 papers \\
\hline \hline
\end{tabular}

높게 나타났다. 이들은 그룹 B의 논문들을 이어주는 영향력 높은 핵심어가 된다.

다음으로 높은 근접 중심성을 보이는 extreme value index(V49)를 중심으로 asymptotic normality(V15), second order parameter(V62), extreme value distribution(V63) 등이 그룹 A의 핵심어 네트워크 를 구성하고, Yun (2005), Yun (2006) 등 11편의 논문에서 핵심어를 공유하고 있다. 그룹 A에서 매개 중심성이 가장 높은 핵심어는 efficiency(V3)이고, consistency(V2), asymptotic normality(V15), coefficient of variation(V7)이 매개 중심성이 높게 나타났다. 이들은 그룹 A의 여러 논문들을 이어주는 영향 


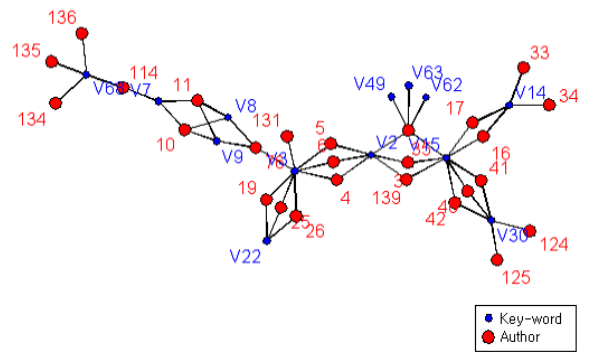

(a) Networks

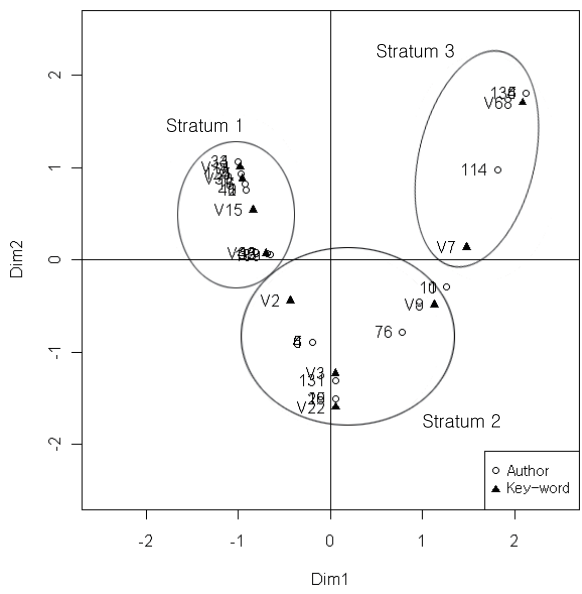

(b) Correspondence analysis plot

Figure 3: Key-words and authors networks, and the correspondence analysis plot by group A

Table 4: Group A of the authors, and key-words for each stratum

\begin{tabular}{ccl}
\hline \hline Stratum & Division & \multicolumn{1}{c}{ Authors and key-words } \\
\hline \multirow{2}{*}{1} & $\begin{array}{c}\text { Authors } \\
\text { Key-words }\end{array}$ & $\begin{array}{l}\text { Yoo Sung Park(19), Sei Jung Park(125), Joong Jae Cho(139) } \\
\text { asymptotic normality(V15), robust(V30), probability of correct selection(V14) }\end{array}$ \\
\hline \multirow{2}{*}{2} & $\begin{array}{c}\text { Authors } \\
\text { Key-words }\end{array}$ & $\begin{array}{l}\text { Dong Hee Lee(131), Byung Jin Choi(10), Kee Young Kim(11) } \\
\text { efficiency(V3), consistency(V2), likelihood ratio test(V8), Wald test(V9) }\end{array}$ \\
\hline \multirow{2}{*}{3} & $\begin{array}{c}\text { Authors } \\
\text { Key-words }\end{array}$ & $\begin{array}{l}\text { Yong Hee Lee(114), Myoung Shic Jhun(135), Jae Won Lee(136) } \\
\text { coefficient of variation(V7), bootstrap(V68) }\end{array}$ \\
\hline \hline
\end{tabular}

력 높은 핵심어가 된다.

높은 근접 중심성을 보이는 rate of convergence(V35)를 중심으로 jump size(V33), one-side ker$\operatorname{nel}(V 34)$ 등이 그룹 C의 핵심어 네트워크를 구성하고, Huh (2004), Kang과 Huh (2006) 등 10편 의 논문에서 핵심어를 공유하고 있다. 그룹 $\mathrm{C}$ 에서 매개 중심성이 가장 높은 핵심어는 weak convergence(V19)이고, 그룹 C에서 여러 논문들을 이어주는 영향력 높은 핵심어가 된다.

핵심어 네트워크에서 형성된 세 그룹의 네트워크 특징을 자세히 살펴보기 위하여, 세 그룹의 저 자와 핵심어에 대한 대응분석을 이용하였다. 먼저 그룹 $\mathrm{A}$ 의 핵심어와 저자 네트워크 그림 및 대응분 석도는 Figure 3의 (a)와 (b)이고, Figure 3의 (a)에서 매개 중심성이 높았던 efficiency(V3)와 consistency(V2)가 두 부분을 이어주는 것을 확인할 수 있다. Figure 3의 (b)를 살펴보면 대응분석도의 설명 력은 $37.46 \%$ 로 나타났고, 계층 1 (제2사분면), 계층2(제3.4사분면), 계층3(제1사분면)으로 나누어지는 것을 확인할 수 있다. Table 4는 그룹 A의 각 계층에서 근접중심성, 매개중심성이 높게 나타난 저자와 핵심어로 분류하였다. 계층1은 Yoo Sung Park(19), Sei Jung Park(125), Joong Jae Cho(139) 등의 저자 가 asymptotic normality(V15), robust(V30), probability of correct selection(V14) 등의 핵심어를 이용하 여 논문을 공저, 계층2는 Dong Hee Lee(131), Byung Jin Choi(10), Kee Young Kim(11) 등의 저자가 efficiency(V3), consistency(V2), likelihood ratio test(V8), Wald test(V9) 등의 핵심어를 이용하여 논문을 공 저, 계층3은 Yong Hee Lee(114), Myoung Shic Jhun(135), Jae Won Lee(136) 등의 저자가 coefficient of variation(V7), bootstrap(V68) 등의 핵심어를 이용하여 논문을 공저하였다. 그룹 A의 각 계층의 특징을 살펴보면 계층 1 의 경우 상대적으로 근접중심성이 높은 핵심어들이 많이 나타났고, 계층 2 의 경우 상대 


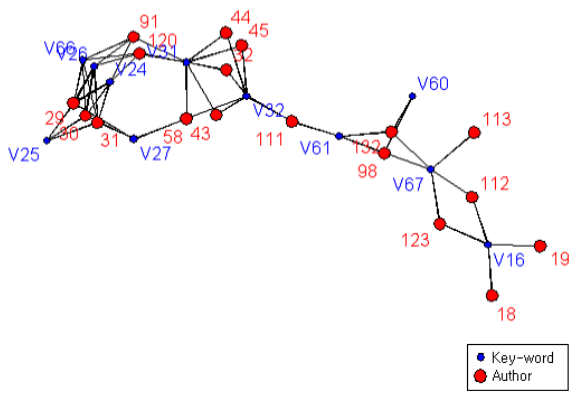

(a) Networks

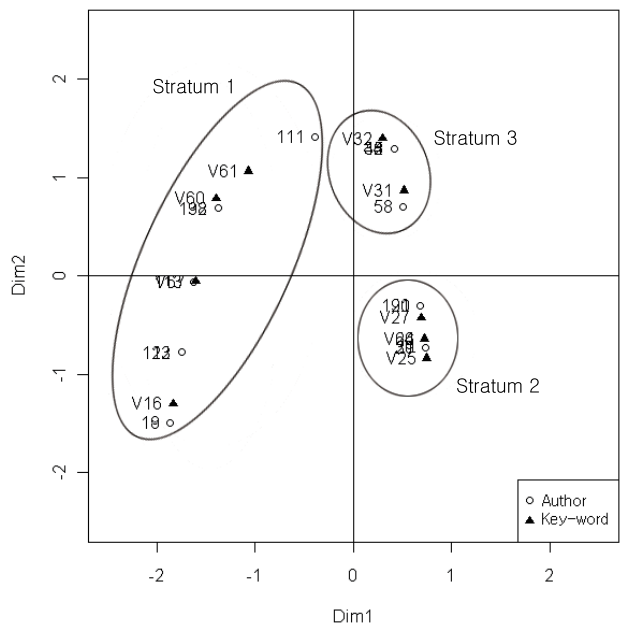

(b) Correspondence analysis plot

Figure 4: Key-words and authors networks, and the correspondence analysis plot by group B

Table 5: Group B of the authors, and key-words for each stratum

\begin{tabular}{|c|c|c|}
\hline Stratum & Division & Authors and key-words \\
\hline \multirow{2}{*}{1} & Authors & Jo Han Lim(112), Seung Jean Kim(113), Jae Yong Lee(98) \\
\hline & Key-words & Markov chain monte carlo(V61), proportional hazard model(V67), variance components(V16) \\
\hline \multirow{2}{*}{2} & Authors & Woo Dong Lee(31), Dal Ho Kim(29), Sang Gil Kang(30) \\
\hline & Key-words & fractional Bayes factor(V27), Matching prior(V24), reference prior(V26) \\
\hline \multirow[t]{2}{*}{3} & Authors & Jung Hoon Jang(45), Tae Young Yang(44), Youn Shik Chung(32) \\
\hline & Key-words & Gibbs sampler(V31), Metropolis-Hastings algorithm(V32) \\
\hline
\end{tabular}

적으로 매개중심성이 높은 핵심어, 계층3의 경우 그 이외의 나머지 핵심어들로 구분되었다.

그룹 B의 핵심어와 저자 네트워크 그림 및 대응분석도는 Figure 4의 (a)와 (b)이고, Figure 4의 (a)에 서 매개 중심성이 높았던 metropolis-hastings algorithm(V32)과 markov chain monte carlo(V61)가 두 부 분을 이어주는 것을 확인할 수 있다. Figure 4의 (b)를 살펴보면 대응분석도의 설명력은 $58.09 \%$ 로 나 타났고, 계층 1 (제 $2 \cdot 3$ 사분면), 계층2(제4사분면), 계층 3 (제 1 사분면)으로 나누어지는 것을 확인할 수 있 다. Table 5는 그룹 B의 각 계층에서 근접중심성, 매개중심성이 높게 나타난 저자와 핵심어로 분류하 였다. 각 계층의 특징을 살펴보면 계층 1 의 경우 상대적으로 매개중심성이 높은 핵심어들이 나타났고, 계층2의 경우 상대적으로 근접중심성이 높은 핵심어, 계층3의 경우 상대적으로 근접중심성과 매개중 심성이 모두 높은 핵심어들로 구분되었다.

그룹 $\mathrm{C}$ 의 핵심어와 저자 네트워크 그림 및 대응분석도는 Figure 5의 (a)와 (b)이고, Figure 5의 (a)에 서 매개 중심성이 높았던 weak convergence(V19)가 세 부분을 이어주는 것을 확인할 수 있다. Figure 5 의 (b)를 살펴보면 대응분석도의 설명력은 $50.31 \%$ 로 나타났고, 계층 1 (제 2 사분면), 계층 2 (제 $3 \cdot 4$ 분면), 계층 3 (제 1 사분면)으로 나누어지는 것을 확인할 수 있다. Table 6 은 그룹 C의 각 계층에서 근접중심성, 매개중심성이 높게 나타난 저자와 핵심어로 분류하였다. 각 계층의 특징을 살펴보면 계층 1 의 경우 상 대적으로 근접중심성과 매개중심성이 높은 핵심어들이 나타났고, 계층2의 경우 상대적으로 근접중심 성이 높은 핵심어, 계층3의 경우 상대적으로 매개중심성이 높은 핵심어들로 구분되었다. 


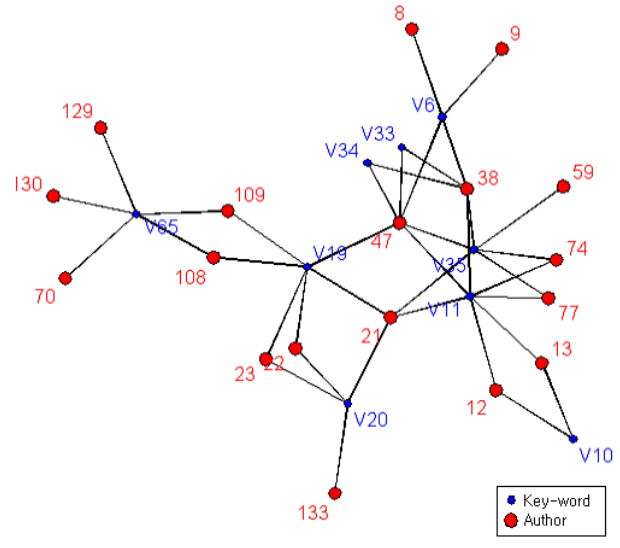

(a) Networks

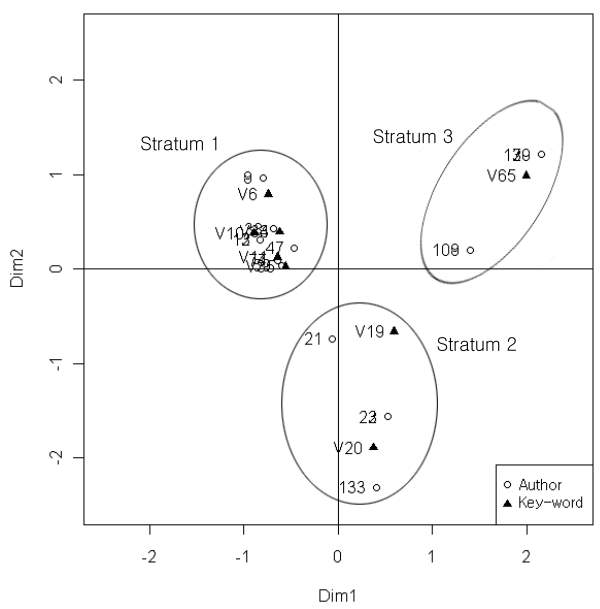

(b) Correspondence analysis plot

Figure 5: Key-words and authors networks, and the correspondence analysis plot by group C

Table 6: Group C of the authors, and key-words for each stratum

\begin{tabular}{|c|c|c|}
\hline Stratum & Division & Authors and key-words \\
\hline \multirow{2}{*}{1} & Authors & Jib Huh(47), Kee Hoon Kang(38), Ta Chen Liang(59) \\
\hline & Key-words & variance estimation(V11), rate of convergence(V35), nonparametric regression(V6) \\
\hline \multirow{2}{*}{2} & Authors & Tae Yoon Kim(21), Tai Sup Lee (23) \\
\hline & Key-words & weak convergence(V19), empirical process(V20) \\
\hline 3 & $\begin{array}{c}\text { Authors } \\
\text { Key-words }\end{array}$ & $\begin{array}{l}\text { Seong Joo Song(108), Per A. Mykland(109) } \\
\text { compound poisson process(V65) }\end{array}$ \\
\hline
\end{tabular}

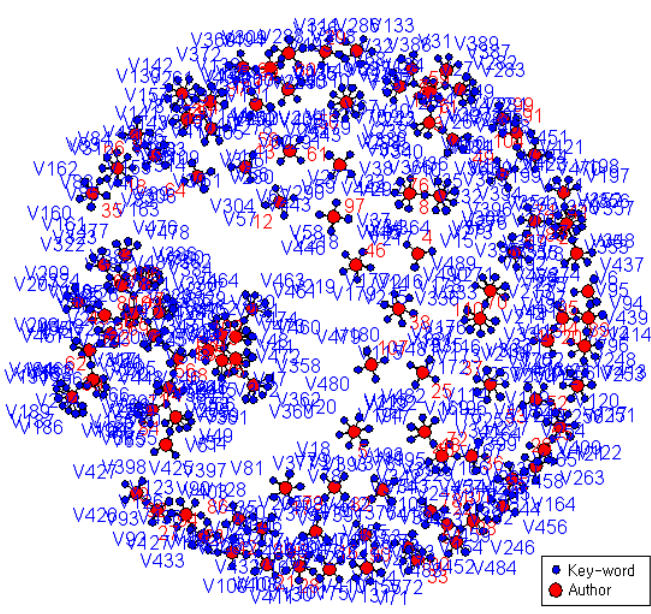

(a) The entire association networks

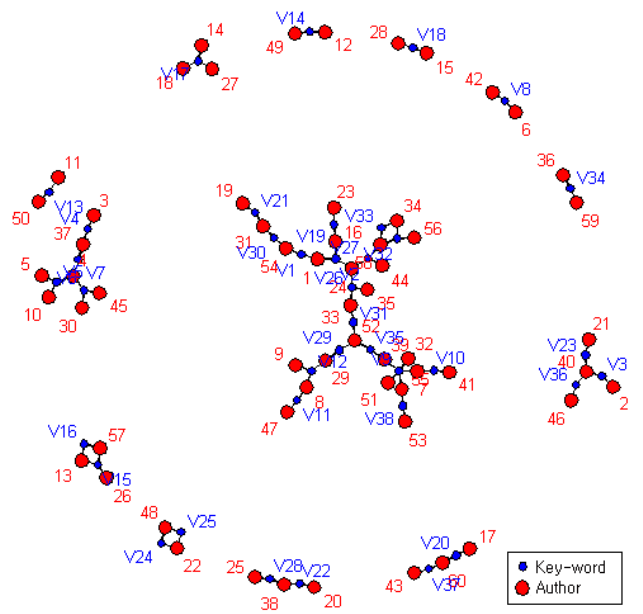

(b) The modified association networks

Figure 6: Papers and key-words associated with the networks after the transition of SCIE 


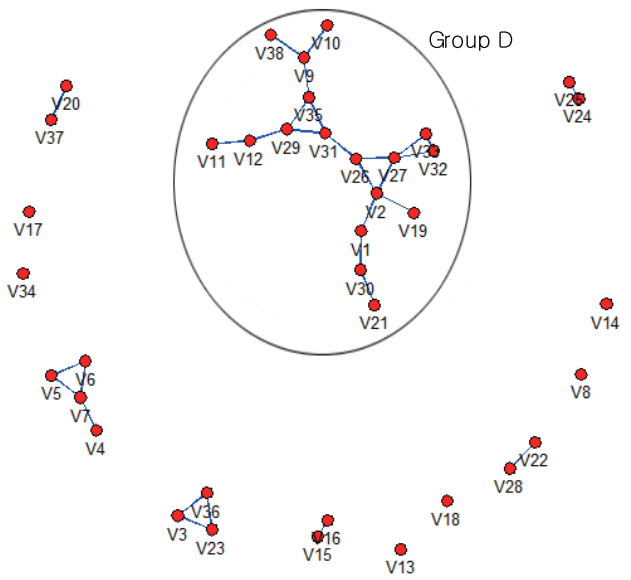

Figure 7: Key-words networks after the transition of SCIE

Table 7: Key-words networks centrality after the transition of SCIE

\begin{tabular}{ccc|ccc}
\hline \hline Key-word & Node & $\begin{array}{c}\text { Closeness } \\
\text { centrality }\end{array}$ & Key-word & Node & $\begin{array}{c}\text { Betweenness } \\
\text { centrality }\end{array}$ \\
\hline maximum likelihood estimator & V2 & 0.2189 & bias & V26 & 0.1922 \\
bias & V26 & 0.2162 & wavelets & V31 & 0.1892 \\
confidence interval & V27 & 0.2122 & maximum likelihood estimator & V2 & 0.1532 \\
wavelets & V31 & 0.2104 & deconvolution & V35 & 0.1171 \\
deconvolution & V35 & 0.1991 & asymptotic normality & V9 & 0.0871 \\
adaptive estimation & V29 & 0.1946 & logistic regression & V1 & 0.0841 \\
empirical likelihood & V32 & 0.1851 & confidence interval & V27 & 0.0841 \\
confidence region & V33 & 0.1851 & adaptive estimation & V29 & 0.0841 \\
\hline \hline
\end{tabular}

Table 8: After the transition of SCIE center of a group of key-words and papers

\begin{tabular}{ccl}
\hline \hline Group & Division & \multicolumn{1}{c}{ Key-words and papers } \\
\hline \multirow{2}{*}{ D } & Closeness centrality & maximum likelihood estimator(V2), bias(V26), confidence interval(V27) \\
& Betweenness centrality & bias(V26), wavelets(V31), maximum likelihood estimator(V2), deconvolution(V35) \\
& Sharing paper & Asgharzadeh (2009), Cavalier and Maimondo (2010), Huang (2010) and 22 papers \\
\hline \hline
\end{tabular}

\section{3. $\mathrm{SCIE}$ 급 전환 이후의 JKSS}

Table 1의 SCIE급 전환 이후의 JKSS를 살펴보면 111 편의 논문, 198 명의 저자, 494 개의 핵심어로 구성되어 있다. Figure 6의 (a)는 논문과 핵심어에 대한 $111 \times 494$ 의 전체 연관네트워크 그림이다. 이 를 살펴보면 SCIE급 전환 이전과 동일하게 방대한 양의 핵심어를 사용함으로 인하여 구체적인 정보를 확인하기 힘들기 때문에 494 개의 핵심어 중에서 최소 2 편 이상의 논문에 제시된 핵심어를 선별하였고, 선별된 38 개의 핵심어가 사용된 60 편의 논문, 107 명의 저자들로 수정된 자료에 대한 분석을 실시하였 다. Figure 6의 (b)는 수정된 $60 \times 38$ 의 연관네트워크 그림이다.

수정된 연관네트워크로 부터 핵심어간의 특징을 살펴보기 위해 행렬교적을 이용한 $38 \times 38$ 인접 행렬의 핵심어 네트워크는 Figure 7과 같다. 식 (2.1)에 의해서 구해진 근접 중심성과 식 (2.2)에 의 해서 구해진 매개 중심성의 값을 기준으로 상위 $20 \%$ 의 노드에 대한 정보는 Table 7 과 같다. 가장 높은 근접 중심성을 보이는 maximum likelihood estimator(V2)를 중심으로 bias(V26), confidence in- 


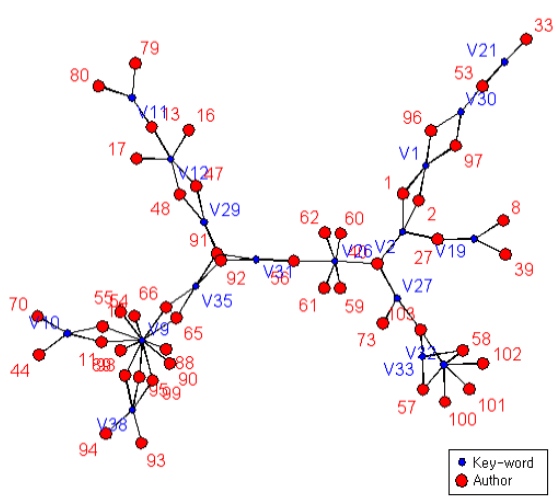

(a) Networks

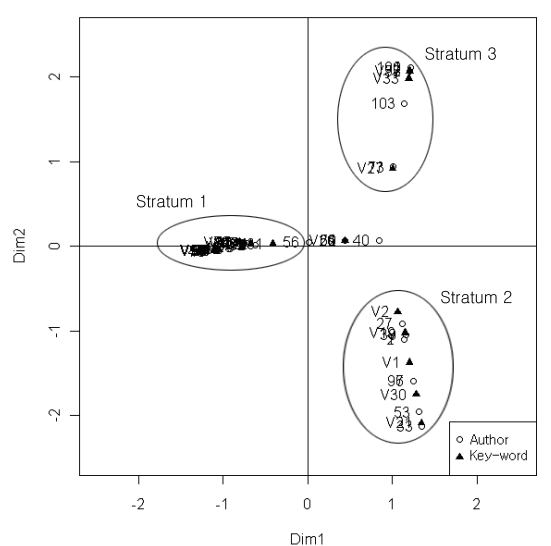

(b) Correspondence analysis plot

Figure 8: Key-words and authors networks, and the correspondence analysis plot by group D

Table 9: Group D of the authors, and key-words for each stratum

\begin{tabular}{ccc}
\hline \hline Stratum & Division & Authors and key-words \\
\hline \multirow{2}{*}{1} & Authors & Laurent Cavalier(91), Marc Raimondo(92), Shota Gugushvili(66), Bert van Es(65) \\
& Key-words & Asymptotic normality(V9), Deconvolution(V35), Wavelets(V31) \\
\multirow{2}{*}{2} & Authors & Dan L. Nicolae(39), Zhensheng Huang(103), Sugata Sen Roy(1), Sibnarayan Guria(2) \\
& Key-words & Maximum likelihood estimator(V2), Logistic regression(V1) \\
\hline \multirow{2}{*}{3} & Authors & Christophe Chesneau(56), Dong Wan Shin(27), Subir Ghosh(96), Hiya Banerjee(97) \\
& Key-words & Confidence interval(V27), Empirical likelihood(V32), Confidence region(V33) \\
\hline \hline
\end{tabular}

terval(V27), wavelets(V31) 등이 하나의 핵심어 네트워크를 구성하고, Asgharzadeh (2009), Cavalier와 Raimondo (2010), Huang (2010) 등 22편의 논문에서 핵심어를 공유하고 있다. 네트워크에서 매개 중 심성이 가장 높은 핵심어는 bias(V26)이고, wavelets(V31), maximum likelihood estimator(V2), deconvolution(V35)이 매개 중심성이 높게 나타났다. 이들은 네트워크에서 여러 논문들을 이어주는 영향력 높은 핵심어가 된다.

핵심어 네트워크에서 형성된 하나의 네트워크의 특징을 자세히 살펴보기 위하여, 저자와 핵심어 에 대한 대응분석을 이용하여 보았다. 그룹 $\mathrm{D}$ 의 핵심어와 저자 네트워크 그림 및 대응분석도는 Figure 8의 (a)와 (b)이고, Figure 8의 (a)에서 매개 중심성이 높았던 Bias(V26)와 Wavelets(V31)가 두 부분을 이어주는 것을 확인할 수 있다. Figure 8 의 (b)를 살펴보면 대응분석도의 설명력은 $20.54 \%$ 로 나타났 고, 계층 1 (제 $2 \cdot 3$ 사분면), 계층2(제4사분면), 계층3(제1사분면)으로 나누어지는 것을 확인할 수 있다. Table 9는 그룹 $\mathrm{D}$ 의 각 계층에서 근접중심성, 매개중심성이 높게 나타난 저자와 핵심어로 분류하였다. 각 계층의 특징을 살펴보면 계층1의 경우 상대적으로 근접중심성과 매개중심성이 높은 핵심어들이 나 타났고, 계층2의 경우 상대적으로 매개중심성이 낮은 핵심어, 계층3의 경우 상대적으로 중개중심성이 높은 핵심어들로 구분되었다.

\section{4. 결론}

본 연구는 사회네트워크 분석을 활용하여 JKSS에 게재된 논문으로부터 핵심어들의 네트워크를 살 펴보고, 더불어 중심성이 높은 핵심어들이 네트워크에서 어떠한 특징을 갖고 있는지 알아보기 위해 대 응분석을 접목시켜 보았다. 분석 결과, SCIE급 전환 이전의 논문과 핵심어 네트워크는 세 그룹의 큰 네 
Table 10: The frequency of personal papers and co-authors

\begin{tabular}{cccccc}
\hline \hline \multirow{2}{*}{ Period } & \multirow{3}{*}{ Personal } & Co-authors & \multicolumn{3}{c}{ Co-authors classified } \\
\cline { 4 - 6 } & & & $\begin{array}{c}\text { National } \\
\text { co-authors }\end{array}$ & $\begin{array}{c}\text { Foreign } \\
\text { co-authors }\end{array}$ & $\begin{array}{c}\text { Domestic and foreign } \\
\text { co-authors }\end{array}$ \\
\hline Before the transition & $33(32.3 \%)$ & $60(67.7 \%)$ & $43(71.7 \%)$ & $8(13.3 \%)$ & $9(15.0 \%)$ \\
After the transition & $19(31.7 \%)$ & $41(68.3 \%)$ & $12(29.3 \%)$ & $26(63.4 \%)$ & $3(7.3 \%)$ \\
\hline \hline
\end{tabular}

트워크와 독립적이거나, 작은 네트워크가 형성되는 특징을 나타내어 세 분야의 연구가 활발히 이루어 진 것을 확인할 수 있었다. 반면에 SCIE급 전환 이후의 논문과 핵심어 네트워크는 하나의 큰 네트워크 와 여러 개의 독립 된 네트워크가 형성되는 특징을 나타내어 특정 한 분야의 연구가 활발히 이루어 진 것을 확인할 수 있었다. 뿐만 아니라 SCIE급 전환 이전의 경우 Table 10 과 같이 60 편의 공저 논문 중 에서 국내저자들의 교류에 의한 논문 투고(43편, $67.7 \%$ )가 많았지만, SCIE급 전환 이후의 경우 41 편의 공저 논문 중에서 국내저자들의 교류에 의한 투고보다는 외국 저자들의 교류에 의한 논문 투고(26편, $63.4 \%)$ 가 많이 증가하였음을 확인할 수 있었다.

\section{References}

Asgharzadeh, A. (2009). Approximate MLE for the scaled generalized exponential distribution under progressive type- II censoring, Journal of the Korean Statistical Society, 38, 223-229.

Barnes, J. (1954). Class and committees in a Norwegian island parish, Human Relations, 7, 39-58.

Cavalier, L. and Raimondo, M. (2010). Multiscale density estimation with errors in variables, Journal of the Korean Statistical Society, 39, 417-429.

Choi, S. B., Kang, C. W., Choi, H. J. and Kang, B. Y. (2011). Social network analysis for a soccer game, Journal of the Korean Data \& Information Science Society, 22, 1053-1063.

Choi, Y. S. (2001). Understanding and Application of Correspondence Analysis using SAS, Freedom Academy, Seoul.

Heo, J. E. and Kim, Y. H. (2007). Developing noninformative priors for the familial data, Journal of the Korean Statistical Society, 36, 77-91.

Huang, Z. (2010). Empirical likelihood for single-index varying-coefficient models with right-censored data, Journal of the Korean Statistical Society, 39, 533-544.

Huh, J. (2004). Nonparametric discontinuity point estimation in generalized linear model, Journal of the Korean Statistical Society, 33, 59-78.

Huh, M. H. (2010). Introduction to Social Network Analysis using R, Freedom Academy, Seoul.

Jung, M. S. and Chung, D. J. (2008). Co-author and keyword networks and their clustering appearance in preventive medicine fields in Korea: Analysis of papers in the Journal of Preventive Medicine and Public Health, 1991 2006, The Korean Society for Preventive Medicine, 41, 1-9.

Kang, K. H. and Huh, J. (2006). Nonparametric estimation of the variance function with a change point, Journal of the Korean Statistical Society, 35, 1-23.

Kim, D. H., Kang, S. K. and Lee, W. D. (2003). Bayesian test for the equality of the means and variances of the two normal populations with variances related to the means using moninformative priors, Journal of the Korean Statistical Society, 32, 271-288.

Kim, D. H., Lee, W. D. and Kang, S. G. (2006). Objective bayesian approach to step stress accelerated life tests, Journal of the Korean Statistical Society, 35, 225-238.

Kim, J. K., Choi, I. Y., Kim, H. K. and Kim, N. H. (2009). Social network analysis to analyze the purchase behavior of chuning customers and loyal customers, The Korean Operations Research and Management Science Society, 26, 183-196. 
Korean Statistical Society (2011). The Korean Statistical Society 40 Years of History, Hanrimwon, Seoul. Sohn, D. W. (2002). Social Network Analysis, Kyungmoon, Seoul.

Yun, S. H. (2005). Estimation of the second order parameter characterizing the tail behavior of probability distributions: Consistency, Journal of the Korean Statistical Society, 34, 273-280.

Yun, S. H. (2006). Estimation of the second order parameter characterizing the tail behavior of probability distributions: Asymptotic normality, Journal of the Korean Statistical Society, 35, 91-103. 